\title{
A comprehensive cerebrovascular simulation model for teaching and research
}

\author{
M. Ursino ${ }^{1}$, M. Giannessi ${ }^{1}$ \& W. B. Murray ${ }^{2}$ \\ ${ }^{1}$ Department of Electronics, Computer Science and Systems, \\ University of Bologna, Italy \\ ${ }^{2}$ Simulation Development and Cognitive Science Laboratory, \\ Penn State University College of Medicine, PA, USA
}

\begin{abstract}
A comprehensive mathematical model of cerebral hemodynamics, with separate regulation of multiple brain regions, is presented. The main sectors included in the model are: the Circle of Willis, the hemodynamic circulation in the six districts perfused by the cerebral arteries, venous return with collapsible veins, cerebrospinal fluid circulation, and intracranial elasticity. Furthermore, each cerebral district is independently regulated following changes in cerebral perfusion pressure and $\mathrm{CO}_{2}$ tension. In this work, the model is used to analyze cerebral hemodynamics during unilateral stenosis or occlusion of an internal carotid artery, as well as to assess the compensatory role of the Circle of Willis and of cerebrovascular regulatory mechanisms. Results show that, in normal subjects, the action of local blood flow regulation mechanisms and compensation by the Circle of Willis ensure adequate ipsilateral blood flow even in the presence of total unilateral internal carotid artery occlusion. However, a steal phenomenon is observed (i.e., a decrease of blood flow in the ipsilateral region) following hypercapnia. Sensitivity analysis on the calibre of the communicating arteries reveals that the anterior communicating artery plays a pivotal role in ensuring blood flow in the ipsilateral side during internal carotid artery stenosis. The model may have important clinical and educational applications, allowing the assessment of blood perfusion to different brain regions in various pathophysiological cases.

Keywords: mathematical model, simulation, cerebral physiology model, circle of Willis, cerebral autoregulation, $\mathrm{CO}_{2}$ reactivity, stroke.
\end{abstract}




\section{Introduction}

The mathematical relationships between cerebral perfusion pressure (CPP), intracranial pressure (ICP) and cerebral blood flow (CBF) as well as the autoregulatory control of these quantities by the normal brain, have been well described [1]. However, the alterations occurring in these quantities in pathological conditions, and their complex non-linear relations are difficult to be understood in simple qualitative terms, and these have not yet been described in a single comprehensive model.

In past years, we developed a comprehensive mathematical model of the cerebral circulation, which included many different aspects of physiopathological relevance: cerebral autoregulation, cerebrospinal fluid (CSF) circulation, $\mathrm{CO}_{2}$ reactivity, intracranial compliance, intracranial pressure (ICP) dynamics and venous collapsibility. With that model, we were able to simulate several physiological phenomena and clinical results on human patients [2].

The previous model, however, included just a single blood pathway inside the brain, i.e., it assumed no difference in cerebral hemodynamic among the different brain regions. For this reason, that model was not able to simulate conditions characterized by regional differences in brain circulation, such as those occurring during occlusion or stenosis of an intracranial vessel, during ischemia in a brain territory, or as a consequence of regional differences in metabolism. In these conditions, the situation is made particularly complex by two phenomena: $\mathrm{CBF}$ regulation mechanisms work in a different way on the individual districts, depending on the local value of blood flow; the different districts communicate upstream via the Circle of Willis, and, although to a lesser extent, via downstream anatomical collaterals.

Hence, the present work was designed with two main purposes:

i) to develop and implement a more complete version of our cerebrovascular model, which describes hemodynamics and blood flow regulation in the six main brain territories separately, and accounts for the compensatory role of the circle of Willis;

ii) to discuss the role of such an extended model, not only in physiological research, but also in clinical practice and medical education.

The model is able to simulate cerebral hemodynamics and ICP changes in different clinical conditions, and in response to various manoeuvres. However, for the sake of brevity, results in this study are concentrated only on the effect of a stenosis or occlusion of one internal carotid artery (ICA), and on the compensatory role of the Circle of Willis.

\section{Method}

The main aspects of the model are briefly summarized below.

\subsection{The large artery circulation}

Hemodynamics in the large intracranial arteries is reproduced through the arrangement of several resistances and compliances. 
i) A first group represents circulation from the extracranial arteries (i.e., the internal carotid arteries, ICAs, and the basilar artery, BA) down to and including the Circle of Willis.

ii) A second group mimics the resistances of the Circle of Willis, that is precommunicating portions of the anterior cerebral arteries $\left(\mathrm{ACA}_{1} \mathrm{~s}\right)$, the anterior communicating artery $(\mathrm{ACoA})$, the precommunicating portions of the posterior cerebral arteries $\left(\mathrm{PCA}_{1} \mathrm{~s}\right)$ and the posterior communicating arteries (PCoAs).

iii) Finally, a third group of resistances simulates the cerebral arteries down to the pial circulation (the postcommunicating portions of the posterior cerebral arteries, the postcommunicating portions of the anterior cerebral arteries, and the middle cerebral arteries; i.e. $\mathrm{PCA}_{2} \mathrm{~s}, \mathrm{ACA}_{2} \mathrm{~s}$ and $\mathrm{MCAs}$ respectively).

\subsection{The pial artery circulation}

The pial arterial-arteriolar circulation is described through six distinct vascular beds. They represent peripheral hemodynamics in the districts supplied by the MCAs, the anterior cerebral arteries (ACAs) and the posterior cerebral arteries (PCAs) respectively. Moreover, the districts are connected through distal cortical anastomoses.

The pial arterial-arteriolar circulation in each district is described by means of a compliance, which accounts for the amount of blood volume stored in the vessels, and a resistance, which describes viscous energy losses. Both parameters are actively modified by the action of cerebrovascular regulatory mechanisms $[3,4]$.

In the model, two main regulatory mechanisms work on the pial circulation, i.e., $\mathrm{CO}_{2}$ reactivity and autoregulation. During hypercapnia, $\mathrm{CO}_{2}$ reactivity causes vasodilation and autoregulation causes a reduction in local blood flow. Moreover, the two mechanisms do not superimpose linearly, but their actions are passed through a sigmoidal relationship with upper and lower saturation in order to describe total vasodilation and total vasoconstriction.

Mathematical equations for peripheral circulation and for regulatory mechanism action in the individual districts have the same form as used previously for the overall cerebrovascular bed [2] (but with different parameter values).

\subsection{The post-capillary circulation}

For the sake of simplicity, the model includes only a single venous pathway from cerebral capillaries down to the dural sinuses, i.e., no distinction is made between the venous circulation in different intracranial districts. This choice is justified by the presence of wide collateral routes among venous circulation in the different districts [5]. The cerebral venous circulation includes:

i) the resistance and the compliance of the pial venules and of proximal veins

ii) a separate description for the resistance of the terminal intracranial veins (lateral lakes and bridge veins) (see [6]). 


\subsection{The cerebrospinal fluid (CSF) circulation and the intracranial pressure}

CSF is produced at the cerebral capillaries and is reabsorbed at the dural sinuses. Both processes are modelled as passive and unidirectional. The ICP value (which represents the external pressure for all intracranial vessels) is computed [6] by applying the mass-preservation principle (including arterial cerebral blood volume, venous cerebral blood volume and CSF volume) and assuming a monoexponential pressure-volume relationship for the craniospinal system [7].

\subsection{Parameter assignment}

All the parameters and input quantities in the model were given values taken from the research (animal and/or human clinical) literature [8-11], in order to reproduce cerebral hemodynamics and intracranial dynamics of a normal subject. Input quantities are arterial pressure, sinus venous pressure and arterial $\mathrm{CO}_{2}$ partial pressure.

\section{Results}

Simulations have been performed by assuming an occlusion or stenosis of one ICA. The stenosis, as modelled, develops along the overall vessel length. The value of resistance of the ICA has been computed, as a function of inner radius reduction, by using Poiseuille's law.

\subsection{The effect of ICA occlusion on the regulatory capacity of the downstream cerebrovascular bed}

The idea is that during a stenosis (or after an occlusion) of one ICA, the downstream vascular bed becomes vasodilated due to autoregulation (which works to maintain local blood flow). Therefore, due to already being vasodilated, the downstream vascular bed cannot respond further to vasodilatory stimuli. In order to test this aspect, we mimicked cerebral hemodynamics assuming different degrees of reductions of the ICA inner radius and, at each level of stenosis, we performed an acute hypercapnia episode (i.e. a step increase in arterial $\mathrm{CO}_{2}$ pressure from 40 to $60 \mathrm{mmHg}$ ). Results are shown in fig. 1 .

In this figure we display the time pattern of blood flow in the ipsilateral and contralateral ICAs (left panels) and blood flow in the ipsilateral and contralateral MCAs (right panels) before hypercapnia and in the 150 seconds following the hypercapnic step. Two aspects of this figure deserve a comment. Firstly, the basal levels of blood flow in the ipsilateral ICA and in the ipsilateral MCA are progressively reduced with the increasing severity of the stenosis. However, even in the presence of total occlusion, blood flow in the MCA is sufficient to maintain metabolism. This is the result of two compensatory mechanisms: the Circle of Willis, which conducts a certain amount of blood flow to the ipsilateral regions, and autoregulation, which vasodilates the ipsilateral micro-vessels. Secondly, the vasodilatory response to $\mathrm{CO}_{2}$ in the ipsilateral side is progressively lost with increasing severity of the stenosis, but the $\mathrm{CO}_{2}$ response is preserved in 
the contralateral side. The model did show a "steal phenomenon" during total occlusion: blood flow in the ipsilateral MCA territory decreases during hypercapnia, since it is stolen by massive vasodilation occurring in the contralateral side.
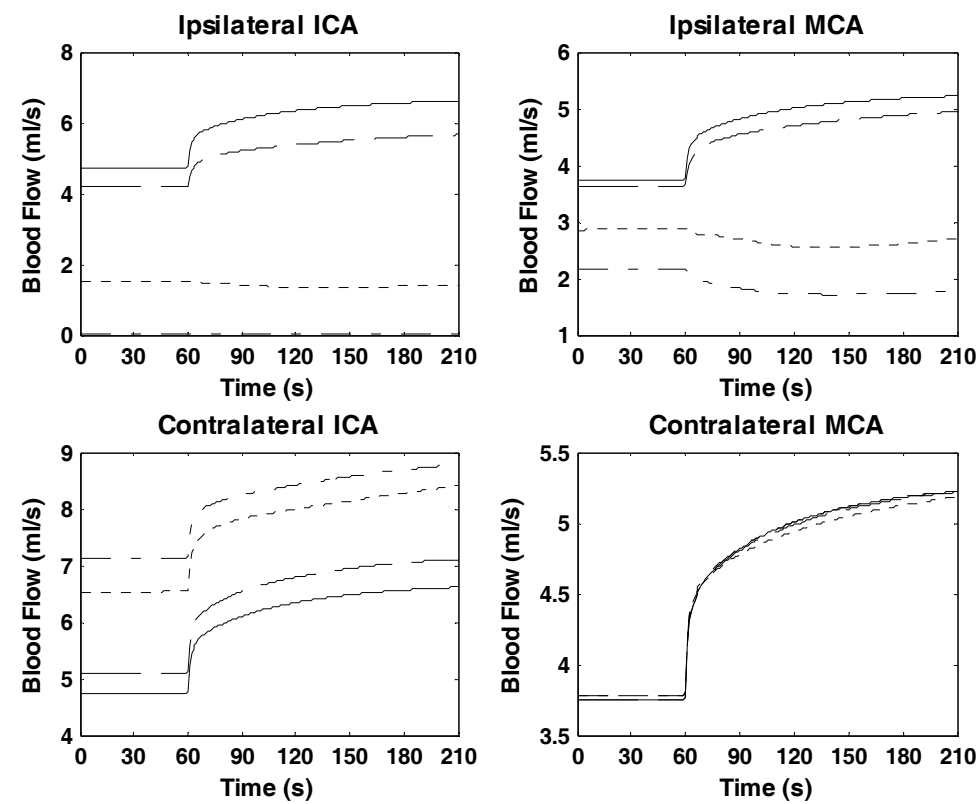

Figure 1: Time pattern of blood flow in the ipsilateral and contralateral ICAs (left panels), and in the ipsilateral and contralateral MCAs (right panels), following a step increase in arterial $\mathrm{CO}_{2}$ pressure from 40 to $60 \mathrm{mmHg}$, performed at time $60 \mathrm{~s}$. Four cases are shown, characterized by different stenosis of the ICA (caliber reduction 90\%: dash-dotted line; 60\%: dotted line; 30\%: dashed line; no reduction: continuous line). It is worth noting the reduction in ipsilateral MCA blood flow during hypercapnia in case of severe stenosis ("steal phenomenon").

\subsection{The compensatory role of the Circle of Willis}

A second set of simulations was performed to assess the role of the posterior and anterior communicating arteries on the blood flow levels during ICA occlusion. To this end, we computed the values of blood flow in all main intracranial arteries during total unilateral ICA occlusion, first by reducing the inner radius of the PCoAs (fig. 2) and then reducing the inner radius of the ACoA (fig. 3). The objective is to evaluate the impact of a reduction in the caliber of communicating arteries (either due to progressive occlusion or individual variability) on the compensatory efficacy of the Circle of Willis. The model demonstrates that, in 
normal conditions, the ACoA plays the major compensatory role during unilateral ICA occlusion. In fact, most of the blood flow reaching the ipsilateral MCA comes from the ACoA via the contralateral ICA, whereas only a minimal amount of blood flow arrives from the PCoAs through the BA. As a consequence, a caliber reduction of both PCoAs has only a negligible detrimental effect (fig. 2).
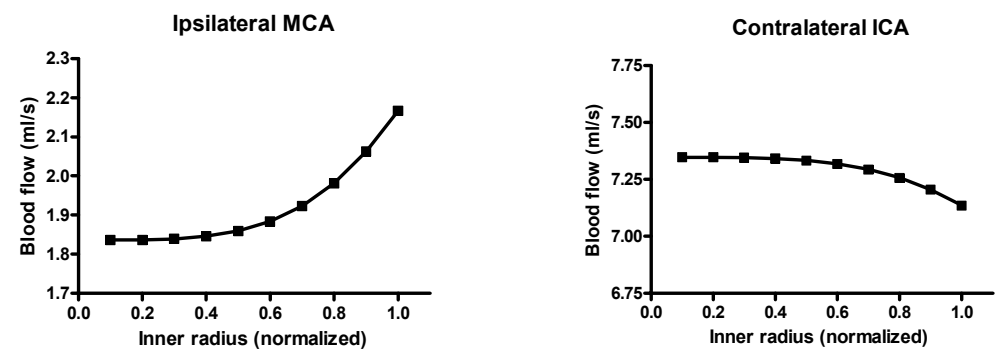

BA
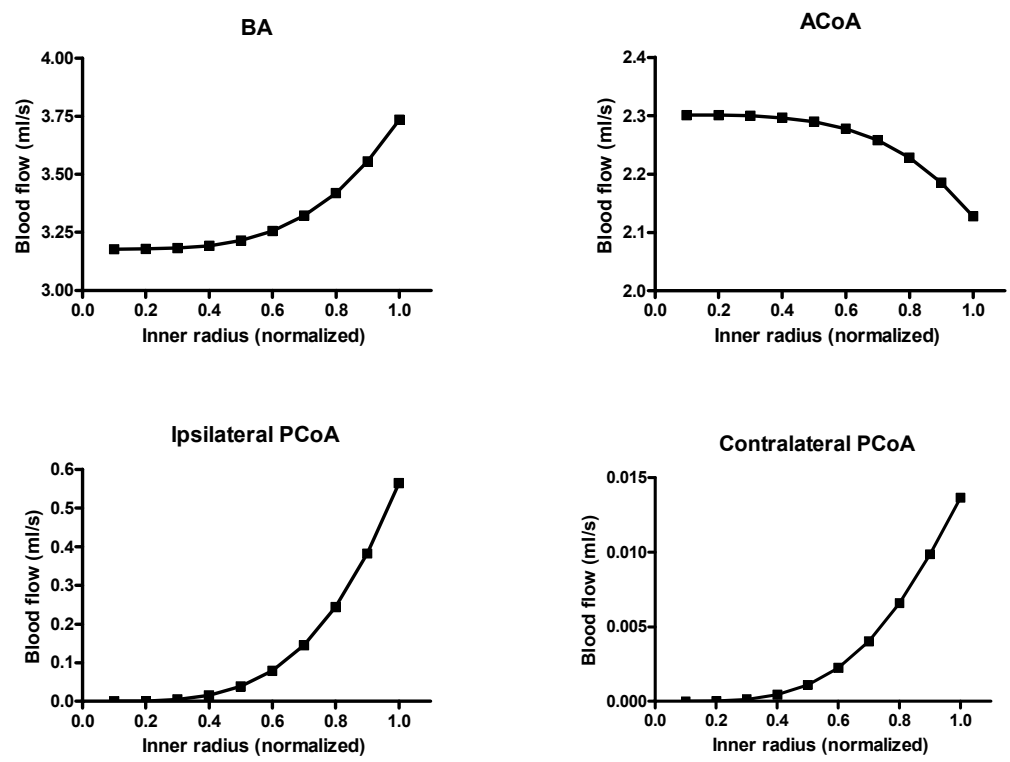

Figure 2: Blood flow in the main intracranial arteries, computed in steady state conditions in a case of total obstruction of one ICA, assuming a progressive reduction in the caliber of both PCoAs. It is worth noting the mild role of these arteries during ICA occlusion.

By contrast, a reduction in the caliber of the ACoA has dramatic consequences, and can only moderately be compensated for an increase in blood through the PCoAs via the basilar artery (fig. 3). We can expect that the role of 
the PCoAs becomes more evident during occlusion of the basilar artery (which is, however, an episode with less clinical impact) or if these vessels exhibit an increase in their caliber (which might occur due to individual variability).

Ipsilateral MCA

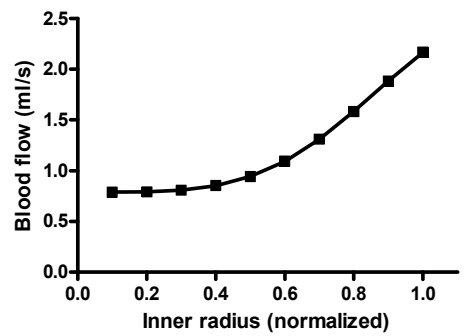

BA

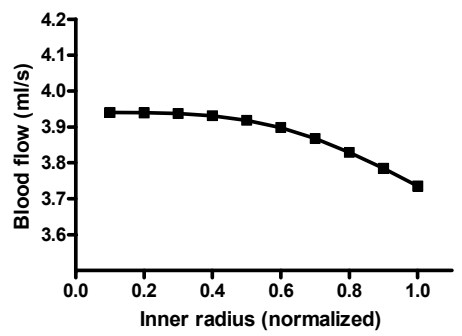

Ipsilateral PCoA



Contralateral ICA

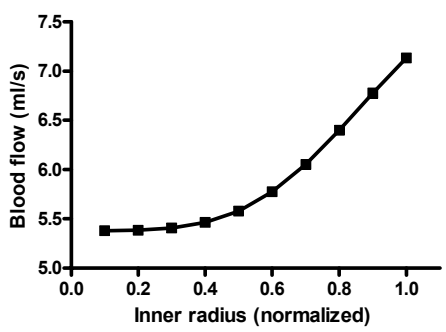

ACoA

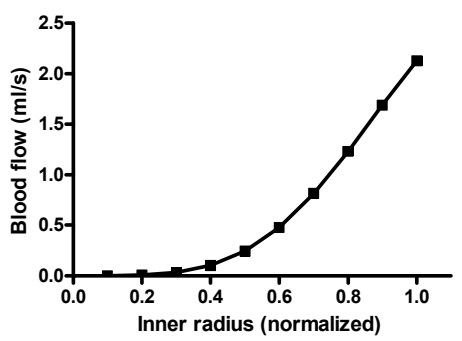

Contralateral PCoA

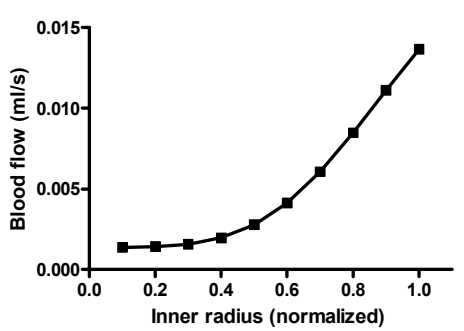

Figure 3: Blood flow in the main intracranial arteries, computed in steady state conditions in a case of total obstruction of one ICA, assuming a progressive reduction in the caliber of the ACoA. It is worth noting the pivotal role played by this artery in maintaining MCA blood flow. 


\section{Discussion}

The mathematical model presented in this work can be used for different purposes: physiological, clinical and educational. In physiological research, the model can be used to assess the role of the main mechanisms involved in cerebral hemodynamics, and to determine their complex relationships in different conditions. The clinical use of the model is especially relevant to the problem of parameter estimation (or parameter assignment). The same model, with different parameter values, can be used to mimic cerebral hemodynamics alterations in different individuals, to analyze the effect of a therapy, and to follow temporal alterations connected with parameter derangements. The educational role of the model is of the greatest value, and is providing an increasing impact in the present days of blunt impact trauma (such a motor vehicle crashes). The model offers the possibility to simulate alternative manoeuvres, to demonstrate their effects in different pathological cases, and to simultaneously analyze the pattern of multiple quantities, some of which (like blood flows in individual territories) cannot be easily monitored (and therefore not taught) in vivo. We believe that these kinds of models, which summarize the complexity of multiple physiopathological relationships into a coherent unitary scenario, may have an increasing impact in medical education in future years.

The examples illustrated in this work are focused on the effect of a reduction of the diameter (unilateral stenosis or occlusion of the ICA; reduction in the calibre of the communicating arteries). The results underline the impressive capacity of cerebral hemodynamics to maintain adequate blood flow levels in peripheral territories despite extreme parameter derangements (like a total unilateral occlusion of the ICA). However, results also emphasize the extreme complexity of the relationships involved, and the possible contradictory effects of the same manoeuvre, depending on parameter values. The "steal phenomenon", illustrated in fig. 1 is a good example of this complexity, and of the dangerous effect that a wrong manoeuvre can have in specific conditions. Hypercapnia, which is often used to vasodilate the cerebrovascular bed and increase cerebral blood flow in patients at risk of ischemia, may have a detrimental effect in patients with ICA occlusion. The model ascribes (and quantifies) the steal phenomenon (well documented in the clinical literature [12, 13]) to an existing previous massive vasodilation in the territory downstream the occluded ICA, caused by blood flow regulatory mechanisms. As a consequence, these vessels have already exhausted their vasodilatory reserve and cannot vasodilate further during hypercapnia. In contrast, contralateral vessels vasodilate during hypercapnia. This vasodilation causes a reduction in arteriolar pressure at the contralateral side, with the genesis of a pressure gradient from the ipsilateral to the contralateral territories.

Simulations on the compensatory capacity of the Circle of Willis stress the pivotal role played by the $\mathrm{ACOA}$, and the strong dependence of cerebral hemodynamics on the calibre of this vessel during ICA obstruction. Hence, patients with a poor anastomotical capacity of the ACoA are at high risk of ischemia during pathologies, which progressively reduce the ICA calibre. 
The cases illustrated here are just a few focused examples of the possibilities offered by the model to help clinical decisions. Additional manoeuvres (not presented for briefness) may include occlusion of a MCA with consequent ischemia of a brain territory, intracranial haemorrhage (either at the periphery, or caused by rupture of an intracranial artery), the effect of head-up tilting, venous obstruction, etc. Moreover, all these manoeuvres may be simulated with normal parameter values, or assuming more severe pathological scenarios (like headinjury, or post-ischemic events) characterized by alterations in autoregulation, CSF circulation, and intracranial elastance.

\section{Conclusion}

This comprehensive cerebrovascular model can represent a valid kernel for building sophisticated innovative medical educational tools, improve clinical understanding when modelled to replicate an individual patient, as well as provide questions for further research.

\section{References}

[1] Ursino, M. \& Lodi, C.A., Interaction among autoregulation, $\mathrm{CO}_{2}$ reactivity, and intracranial pressure: a mathematical model. Am J Physiol 274 (Heart Circ Physiol 43): H1715-H1728, 1998.

[2] Ursino, M., Ter Minassian, A., Lodi, C.A. \& Beydon, L., Cerebral hemodynamics during arterial and $\mathrm{CO}_{2}$ pressure changes: in vivo prediction by a mathematical model. Am J Physiol Heart Circ Physiol, 279: H2439-H2455, 2000.

[3] Lodi, C.A. \& Ursino, M., Hemodynamic effect of cerebral vasospasm in humans: a modeling study. Ann Biomed Eng, 27: 257-273, 1999.

[4] Ursino, M. \& Lodi C.A., A simple mathematical model of the interaction between intracranial pressure and cerebral hemodynamics. J Appl Physiol, 82: 1256-1269, 1997.

[5] Edvisson, L., MacKenzie, E.T. \& McCulloch, J., Cerebral Blood Flow and Metabolism, Raven Press: New York, 1993.

[6] Ursino, M. \& Di Giammarco, P., A mathematical model of the relationship between cerebral blood volume and intracranial pressure changes. The generation of plateau waves. Ann Biomed Eng, 19: 15-42, 1991.

[7] Avezaat, C.J.J., van Eijndhoven, J.H.M. \& Wyper, D.J., Cerebrospinal fluid pulse pressure and intracranial volume-pressure relationship. $J$ Neurol Neurosurg Psychiatry, 42: 687-700, 1979.

[8] Gibo, J.M., Lenkey, C. \& Rhoton, A.L., Microsurgical anatomy of the supraclinoid portion of the internal carotid artery. J Neurosurg, 55: 560574, 1981.

[9] Gilsbach, J.M., Intraoperative Doppler Sonography in Neurosurgery, Springer: Wien, 1983. 
[10] Lindegaard, K.F., Bakke, S.J., Grolimund, P., Aaslid, R., Huber, P. \& Nornes, H., Assessment of intracranial hemodynamics in carotid artery disease by transcranial Doppler ultrasound. J Neurosurg, 63: 890-898, 1985.

[11] Perlmutter, D. \& Rhoton, A.L., Microsurgical anatomy of the anterior cerebral - anterior communicating - recurrent artery complex. $J$ Neurosurg, 45: 259-271, 1976.

[12] Furst, H., Hartl, W.H. \& Janssen, I., Patterns of cerebrovascular reactivity in patients with unilateral asymptomatic carotid artery stenosis. Stroke, 25(6): 1193-1200, 1994.

[13] Baumgartner, R.W. \& Baumgartner, I., Vasomotor reactivity is exhausted in transient ischaemic attacks with limb shaking. J Neurol Neurosurg Psychiatry, 65: 561-564, 1998. 